\title{
REDUÇÃO CIRÚRGICA DA LUXAÇÃO DO QUADRIL EM PACIENTES COM ARTROGRIPOSE MÚLTIPLA CONGÊNITA - ACESSO ANTEROMEDIAL
}

\author{
OPEN REDUCTION OF HIP DISLOCATION IN PATIENTS WITH ARTHROGRYPOSIS \\ MULTIPLEX CONGENITA- AN ANTEROMEDIAL APPROACH
}

Luis Eduardo Munhoz da Rocha ${ }^{2}$, Fábio Koiti Nishimori ${ }^{3}$, Daniel Carvalho de Figueiredo 4

Dulce Helena Grimm², Luiz Antonio Munhoz da Cunha'

\section{RESUMO}

Objetivo: Avaliar os resultados do tratamento cirúrgico da luxação do quadril através do acesso anteromedial em pacientes com artrogripose múltipla congênita (AMC). Métodos: Retrospectivamente foram revisados os prontuários e radiografias de sete crianças com AMC que apresentavam luxação do quadril, totalizando 10 quadris luxados. Foi avaliada a mobilidade articular pré e pós-operatória através da somatória do arco de mobilidade articular em flexão e abdução. Radiograficamente foram avaliados, no pré-operatório, o ângulo acetabular e a altura do colo do fêmur e, no pós-operatório, a continuidade do arco de Shenton, ângulo de Sharp e ângulo CE (centro borda). Quando foi identificada a necrose avascular, esta foi classificada segundo Ogden e Bucholz. Resultados: A média de idade das crianças na ocasião da cirurgia era de 5,5 meses (três a 11 meses). O seguimento médio dos pacientes foi de 9,5 anos (dois a 13 anos). A média de amplitude de movimento da somatória do arco de mobilidade articular em flexão e abdução no exame pré-operatório foi de $108^{\circ}\left(70\right.$ a $\left.155^{\circ}\right)$ e no pós-operatório foi de $125^{\circ}\left(75^{\circ}\right.$ a $\left.175^{\circ}\right)$. Na última avaliação, oito quadris estavam centrados e dois subluxados. Dois quadris foram submetidos a uma osteotomia de ilíaco do tipo Salter. Dois quadris apresentaram sinais significativos de necrose avascular Ogden tipo IV. Oito quadris foram considerados como bons resultados e dois como regulares. Conclusão: Consideramos a via anteromedial uma boa opção para tratamento da luxação de quadril em pacientes de baixa idade com artrogripose múltipla congênita.

Descritores - Artrogripose; Luxação do quadril; Procedimentos cirúrgicos operatórios

\section{ABSTRACT}

Objective: To evaluate the results of the surgical treatment of hip dislocation through the anteromedial approach in patients with arthrogryposis multiplex congenita (AMC). Methods: A retrospective review of the charts and radiographs of 7 children with AMC that presented dislocation of the hiprevised, totalling 10 dislocated hips. : Pre and postoperative articular mobility, was evaluated by summing the joint range-of-motion arc of flexion and abduction., Pre-operatively, the acetabular angle and height of the neck of femur were evaluated radiographically and postoperatively, the continuity of the arc of Shenton, acetabular angle, Sharp angle and CE angle. The presence of avascular necrosis was classified according to Ogden and Bucholz. Results: The average of age of the children at the time of the surgery was 5.5 months (3 to 11 months). The average follow-up time for the patients was 9.5 years (2 to 13 years). The average of the amplitude of movement of the sum of the joint mobility arc in flexion and abduction in the pre-operative examination was $108^{\circ}\left(70^{\circ}\right.$ to $\left.155^{\circ}\right)$ and postoperatively it was $125^{\circ}\left(75^{\circ}\right.$ $175^{\circ}$ ). In the last evaluation, eight hips were centered and two were subluxated. Two hips had been submitted to a Salter iliac osteotomy. Two hips (20\%) had presented significant signs of Ogden type IV avascular necrosis. Eight hips had good results while two were fair. Conclusion: We consider the antero-medial approach a good option for the treatment of the dislocation of the hip in patients of low congenital age with arthrogryposis multiplex congenita.

Keywords - Arthrogryposis; Hip Dislocation; Surgical Procedures, Operative

\footnotetext{
1 - Médico Ortopedista Chefe do Serviço de Ortopedia Pediátrica do Hospital Pequeno Príncipe (HPP) - Curitiba, PR, Brasil.

2 - Médico Ortopedista do Hospital Pequeno Príncipe (HPP) - Curitiba, PR, Brasil.

3 - Médico Residente de Ortopedia e Traumatologia do Hospital Pequeno Príncipe (HPP) - Curitiba, PR, Brasil.

4 - Médico Ortopedista do Hospital Pequeno Príncipe (HPP) - Curitiba, PR, Brasil. Especializando em Ortopedia Pediátrica.
}

Trabalho realizado no Hospital Pequeno Príncipe (HPP) - Curitiba, PR, Brasil.

Correspondência: Fábio Koiti Nishimori - Rua Engenheiro Niepce da Silva, 200, ap. 14, bloco 4 - Portão - 80610-280 - Curitiba PR. - E-mail: fabionishi@gmail.com 


\section{INTRODUÇÃO}

Artrogripose múltipla congênita (AMC) é uma síndrome de etiologia desconhecida, de caráter não progressivo que se caracteriza pela presença de múltiplas contraturas articulares com substituição da musculatura por bandas fibrosas e gordura, deformidade dos membros, geralmente simétrica e inteligência normal ${ }^{(1)}$. Stern, em 1923, foi o primeiro a utilizar o termo artrogripose múltipla congênita, descrevendo quatro casos clínicos e então caracterizando a síndrome ${ }^{(2,3)}$.

O quadril está acometido em 51 a $85 \%$ dos casos de AMC como contraturas fixas e entre 15 a $31 \%$ de luxação ${ }^{(4)}$.

A luxação do quadril artrogripótico é definida como teratológica, portanto, ocorre em uma fase precoce da vida intrauterina. $\mathrm{O}$ acetábulo é pequeno, raso e preenchido por tecido fibroso-gorduroso. A cabeça femoral hipoplásica é frequentemente aplanada na sua porção medial. O ângulo de anteversão pode variar muito, podendo ser retrovertido, em contraste com a luxação típica do quadril em que o ângulo de anteversão está aumentado na grande maioria dos $\operatorname{casos}^{(5,6)}$.

O quadril artrogripótico é caracterizado por diminuição da mobilidade, ausência de função muscular normal e predisposição à perda de mobilidade após o tratamento cirúrgico ${ }^{(5,7,8)}$, características que dificultam a abordagem destes quadris. A redução incruenta geralmente não é efetiva ${ }^{(3,9)}$. Alguns autores recomendam que a luxação bilateral dos quadris fique sem tratamento devido à alta incidência de complicações ${ }^{(6,7,9,10)}$, embora exista controvérsia ${ }^{(11)}$. Nos casos de luxação unilateral, a maioria indica o tratamento cirúrgico com o objetivo de evitar obliquidade pélvica e escoliose ${ }^{(6,7,11-13)}$.

A redução da luxação pelo acesso anterior iliofemoral associado ao encurtamento femoral é o procedimento mais utilizado em crianças acima de 18 meses, embora alguns autores realizem a redução pelo acesso anteromedial em criança de baixa idade, com resultados satisfatórios ${ }^{(4,14)}$.

Este estudo tem como objetivo avaliar os resultados do tratamento cirúrgico com redução pelo acesso anteromedial da luxação do quadril nos pacientes com AMC.

\section{MATERIAIS E MÉTODOS}

Foram revisados 51 prontuários e radiografias de pacientes que apresentavam AMC. Catorze pacientes apresentavam 23 quadris luxados. No período de janeiro de 1990 a janeiro de 2006, 10 quadris luxados em sete crianças foram reduzidos cirurgicamente através do acesso anteromedial no Hospital Pequeno Príncipe.

O diagnóstico de AMC foi realizado segundo os critérios clínicos de Fisher: 1) contraturas articulares ao nascimento, em ao menos duas articulações, exceto o pé torto congênito; 2) inexistência de doença neurológica progressiva; 3 ) evidência de fraqueza muscular com articulações fusiformes. A presença de 'dimples' (prega cutânea), pterígeo ou ausência de pregas cutâneas ${ }^{(1)}$.

Foram objetos deste estudo os casos com o seguimento mínimo de 24 meses e que não haviam sido submetidos a tratamento prévio.

O grau de função não foi utilizado como critério para classificação do resultado; entretanto, todas as crianças foram classificadas no pós-operatório em: deambulador comunitário, deambulador domiciliar, deambulador não funcional ou não deambulador ${ }^{(15)}$. Foi relatada a necessidade do uso de órtese para locomoção.

Os resultados foram avaliados em relação aos dados clínicos e radiográficos.

A avaliação clínica pré e pós-operatória consistiu na somatória do arco de movimento dos quadris em flexão e abdução. Os resultados foram considerados bons, regulares ou ruins conforme o grau de mobilidade articular na última consulta e a presença ou não de subluxação do quadril. (Tabela 1).

Tabela 1 - Tabela de critérios para avaliação dos resultados.

\begin{tabular}{c|c|c}
\hline & Arco de movimento F+ Abd & $\mathbf{R x}$ \\
\hline Bom & $\geq 90^{\circ}$ & Centrado \\
\hline Regular & $60^{\circ}-89^{\circ}$ & Subluxado \\
\hline Ruim & Rigidez & Luxado \\
\hline
\end{tabular}

Na avaliação radiográfica pré-operatória foi medido o ângulo acetabular e a altura do fêmur proximal em relação à linha de Hilgenheiner.

$\mathrm{Na}$ avaliação pós-operatória utilizou-se a medida do ângulo acetabular nas crianças cuja cartilagem trirradiada ainda estivesse aberta e do ângulo de Sharp ${ }^{(16)}$ naquelas que apresentavam a cartilagem trirradiada fechada. Foi avaliada ainda a integridade do arco de Shenton e do ângulo CE de Wiberg ${ }^{(17)}$ nas crianças acima de cinco anos. Foram considerados centrados os quadris em que o arco de Shenton estivesse íntegro e CE maior ou igual a $15^{\circ}$. Necrose avascular foi classificada segundo $\operatorname{Ogden}^{(18,19)}$. 


\section{RESULTADOS}

Dos sete pacientes participantes do trabalho, quatro eram do sexo masculino e três do sexo feminino. Três tinham luxação bilateral dos quadris e quatro unilateral (dois à direita e dois à esquerda).

A média de idade das crianças na ocasião da cirurgia era de 5,5 meses (três a 11 meses). Os pacientes foram imobilizados por um período de seis semanas e uma órtese do tipo Ilfeld foi utilizada em três pacientes após a retirada do gesso (Tabela 2). O seguimento médio foi de 9,5 anos (dois a 13 anos).

A média da amplitude de movimento em flexo-extensão dos quadris no exame pré-operatório foi de $92,5^{\circ}$ $\left(60^{\circ}\right.$ a $\left.135^{\circ}\right)$ e a média da amplitude de movimento em abdução foi de $19^{\circ}\left(0^{\circ}\right.$ a $\left.30^{\circ}\right)$. Na somatória dos movimentos de flexão com abdução no período pré-operatório obtivemos uma média de $108^{\circ}\left(70^{\circ}\right.$ a $\left.155^{\circ}\right)$.

A média de amplitude de movimento no exame pósoperatório em flexo-extensão dos quadris foi de $92^{\circ}\left(50^{\circ}\right.$ a $130^{\circ}$ ), enquanto a média de amplitude de movimento em abdução foi de $33,5^{\circ}\left(10^{\circ}\right.$ a $\left.65^{\circ}\right)$. Na somatória dos movimentos de flexão com abdução no período pós-operatório obtivemos uma média de $125^{\circ}\left(75^{\circ}\right.$ a $\left.175^{\circ}\right)$. Dois quadris apresentavam uma somatória de movimentos entre 60 e $89^{\circ}$ (regulares), oito quadris maior que $90^{\circ}$ (bons) e nenhum caso de rigidez articular (ruim) (Tabela 3 ).

Em relação ao estudo radiográfico, no exame préoperatório foi aferido o ângulo acetabular que teve como média $41^{\circ}\left(28^{\circ}\right.$ a $\left.50^{\circ}\right)$ e oito de 10 quadris se apresentavam com a metáfise proximal do fêmur ao nível da linha de Hilgenheiner.
No pós-operatório, a média do ângulo acetabular foi de $21^{\circ}\left(20^{\circ}\right.$ a $\left.22^{\circ}\right)$ em três quadris. O valor médio do ângulo de Sharp em sete quadris foi de $44,5^{\circ}\left(38^{\circ}\right.$ a $\left.49^{\circ}\right)$. A média do ângulo $\mathrm{CE}$ foi de $26,8^{\circ}\left(7^{\circ}\right.$ a $\left.46^{\circ}\right)$ em sete quadris que apresentavam mais de cinco anos de idade (Tabela 2).

Em relação à função, seis pacientes eram deambuladores comunitários, e um não-deambulador. Três pacientes deambulavam sem órtese e três deambulavam com órtese bilateralmente, sendo cinco do tipo suropodálica e uma do tipo cruropodálica. O paciente que não deambulava fazia uso de tala cruropodálica bilateralmente.

Dos 10 quadris reduzidos por via anteromedial, oito quadris estavam centrados na última consulta e dois estavam subluxados. A necrose avascular tipo IV foi observada em dois quadris, enquanto os outros oito quadris não apresentavam necrose (Tabela 4).

Um paciente apresentou subluxação dos dois quadris (Figura 1). Foi realizada uma osteotomia de ilíaco tipo Salter à direita, dois anos e seis meses após a redução do quadril, e à esquerda dois anos e 11 meses após a redução por via anteromedial (Figura 2). Na última avaliação, o quadril esquerdo estava subluxado (Figura 3) (Tabela 4 - caso dois).

\section{DISCUSSÃO}

A literatura é restrita quanto ao tratamento da luxação de quadril na AMC em paciente abaixo de dois anos de idade, e os trabalhos existentes apresentam um número pequeno de pacientes ${ }^{(8-10,20,21)}$. Os estudos descritos na literatura são, na sua maioria, voltadas ao manejo total do

Tabela 2 - Avaliação dos pacientes segundo número de ordem, iniciais, lado acometido, idade do tratamento, tempo de seguimento, ângulo acetabular pré-operatório, último ângulo acetabular ou ângulo de Sharp, último arco de Shenton quebrado (Q), intacto (I), nível da metáfise proximal do fêmur no pré-operatório, índice CE da última consulta.

\begin{tabular}{|c|c|c|c|c|c|c|c|c|}
\hline Paciente & LA & IDtto & TS & IA Pré & IA/ Sharp(S) último & S Ult & Gage Pré & CE \\
\hline 1. & $\mathrm{D}$ & $6 m$ & $13 A+8$ & $50^{\circ}$ & $S=49^{\circ}$ & $Q$ & 0 & $7^{\circ}$ \\
\hline 2. & E & $6 m$ & $13 A+8$ & $45^{\circ}$ & $S=45^{\circ}$ & I & 0 & $17^{\circ}$ \\
\hline 3. & E & $7 \mathrm{~m}$ & $13 A+2$ & $42^{\circ}$ & $S=48^{\circ}$ & I & 0 & $40^{\circ}$ \\
\hline 4. & $E$ & $11 \mathrm{~m}$ & $5 A+4$ & $36^{\circ}$ & $S=38^{\circ}$ & I & 0 & $0^{\circ}$ \\
\hline 5. & $\mathrm{D}$ & $3 m$ & $9 A+1$ & $40^{\circ}$ & $S=42^{\circ}$ & I & 0 & $46^{\circ}$ \\
\hline 6. & E & $3 m$ & $9 A+1$ & $45^{\circ}$ & $\mathrm{S}=42^{\circ}$ & I & 0 & $46^{\circ}$ \\
\hline 7. & $\mathrm{D}$ & $8 m$ & $14 A+3$ & $28^{\circ}$ & $\mathrm{S}=48^{\circ}$ & I & 0 & $32^{\circ}$ \\
\hline 8. & $\mathrm{D}$ & $4 \mathrm{~m}$ & $2 A+10$ & $33^{\circ}$ & $22^{\circ}$ & I & -1 & - \\
\hline 9. & $\mathrm{E}$ & $4 m$ & $2 A+10$ & $45^{\circ}$ & $20^{\circ}$ & Q & 0 & - \\
\hline 10. & D & $3 m$ & $5 A+4$ & $45^{\circ}$ & $21^{\circ}$ & I & -1 & \\
\hline
\end{tabular}


Tabela 3 - Distribuição segundo iniciais, abdução pré-operatória e última, flexão pré-operatória e última, soma do arco de movimento pré operatório e somatório pós-operatório.

\begin{tabular}{|c|c|c|c|c|c|c|}
\hline Paciente & $A b$ & $A b$ & $\mathbf{F}$ & $\mathbf{F}$ & Somatória & Somatório \\
\hline & Pré & ult & Pré & Ult & Pré-Op & Pós-Op \\
\hline 1. & $10^{\circ}$ & $25^{\circ}$ & $60^{\circ}$ & $50^{\circ}$ & 70 & 75 \\
\hline 2. & $15^{\circ}$ & $30^{\circ}$ & $60^{\circ}$ & $60^{\circ}$ & 75 & 90 \\
\hline 3. & $20^{\circ}$ & $20^{\circ}$ & $90^{\circ}$ & $70^{\circ}$ & 110 & 90 \\
\hline 4. & $30^{\circ}$ & $40^{\circ}$ & $120^{\circ}$ & $110^{\circ}$ & 150 & 150 \\
\hline 5. & $30^{\circ}$ & $40^{\circ}$ & - & $130^{\circ}$ & - & 170 \\
\hline 6. & $30^{\circ}$ & $40^{\circ}$ & - & $130^{\circ}$ & - & 170 \\
\hline 7. & $20^{\circ}$ & $40^{\circ}$ & $135^{\circ}$ & $90^{\circ}$ & 155 & 130 \\
\hline 8. & - & $10^{\circ}$ & - & $70^{\circ}$ & - & 80 \\
\hline 9. & - & $25^{\circ}$ & - & $100^{\circ}$ & - & 125 \\
\hline 10. & $0^{\circ}$ & $65^{\circ}$ & $90^{\circ}$ & $110^{\circ}$ & 90 & 175 \\
\hline Resultados & $19,3^{\circ}$ & $33,5^{\circ}$ & $92,5^{\circ}$ & $92^{\circ}$ & 108,3 & 125,5 \\
\hline
\end{tabular}

Tabela 4 - Distribuição segundo iniciais, registro geral, data de nascimento, sexo, resultado final e classificação de necrose de Ogden.

\begin{tabular}{|c|c|c|c|c|c|}
\hline Paciente & RGT & Nascimento & Sexo & Resultados & Ogden \\
\hline 1. & 5238 & 17/07/1991 & $\mathrm{M}$ & Centrado & 4 \\
\hline 2. & 5238 & 17/07/1991 & $M$ & Subluxado & - \\
\hline 3. & 35305 & 21/01/1992 & M & Centrado & 4 \\
\hline 4. & 57124 & $11 / 03 / 2000$ & $\mathrm{~F}$ & Centrado & - \\
\hline 5. & 47444 & 17/06/1996 & M & Centrado & - \\
\hline 6. & 47444 & 17/06/1996 & $\mathrm{M}$ & Centrado & - \\
\hline 7. & 30116 & $15 / 09 / 1990$ & $M$ & Centrado & - \\
\hline 8. & 2241391 & $30 / 05 / 2003$ & $\mathrm{~F}$ & Centrado & - \\
\hline 9. & 2241391 & $30 / 05 / 2003$ & $\mathrm{~F}$ & Subluxado & - \\
\hline 10. & 57140 & 28/09/1999 & $\mathrm{F}$ & Centrado & - \\
\hline
\end{tabular}

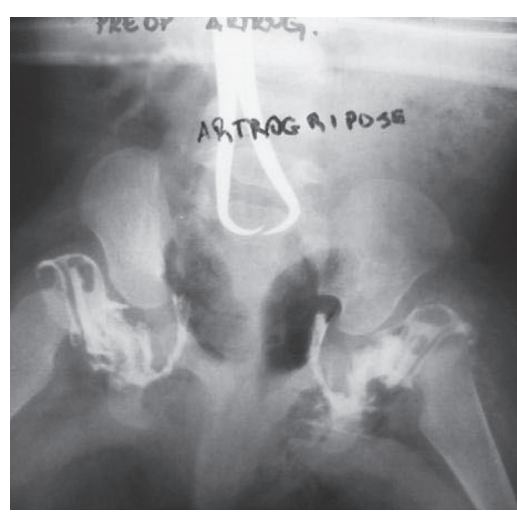

Figura 1 - Casos 1 e 2: artrografia pré-operatória.

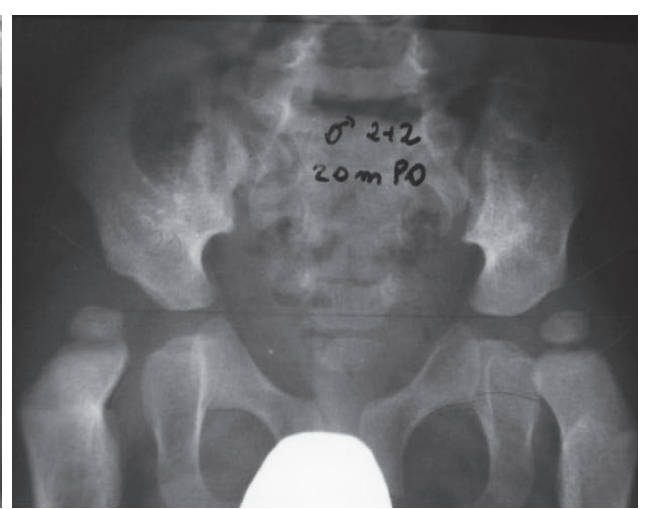

Figura 2 - Caso 1: 20 meses pós-operatório. Subluxação do quadril direito.

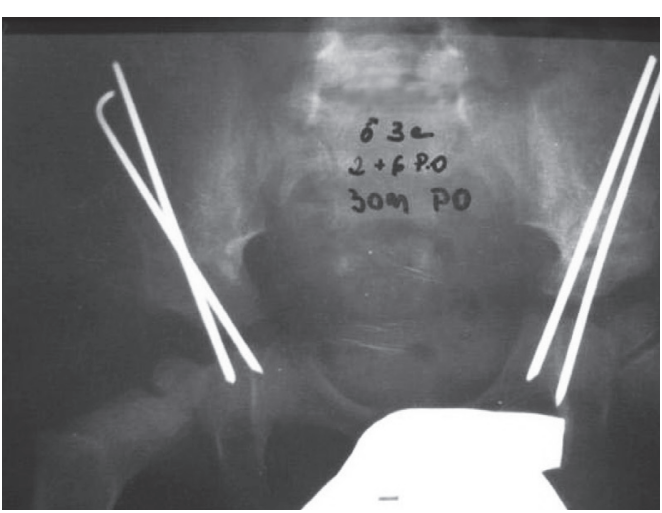

Figura 3 - Casos 1 e 2: 30 meses pós-operatório. Osteotomia de ilíaco tipo Salter bilateral. paciente, e poucas informações específicas e de consenso são obtidas no tratamento da luxação do quadril(22).

Quanto às formas de redução da luxação do quadril, a literatura é consistente, e a maioria dos autores acredita que a redução incruenta não é efetiva ${ }^{(5,8,13,20,23)}$. Gruel et $a l^{(13)}$, em sua série, trataram dois pacientes através de redução incruenta e obtiveram maus resultados, enquanto que os melhores resultados foram obtidos com 
redução cruenta via anterior iliofemoral com encurtamento femoral. Neste estudo não foi utilizada técnica de redução incruenta em nenhum dos quadris luxados.

Da mesma forma em que na luxação congênita do quadril, a idade influencia na escolha da abordagem. Staheli et $a l^{(11)}$ e Herring e Banta ${ }^{(14)}$ defendem que a redução cruenta via anteromedial seja realizada até o sexto mês de vida; entretanto, Coleman ${ }^{(5)}$ refere em seu estudo que a mesma possa ser realizada até o segundo ano de vida.

Nos quadris artrogripóticos a abordagem cirúrgica preconizada acima de dois anos de idade é a redução cirúrgica pelo acesso anterior iliofemoral com encurtamento femoral, com o objetivo de facilitar a redução e diminuir a incidência de necrose avascular ${ }^{(3)}$.

Os relatos de literatura mostram a relativa frequência da necessidade de procedimentos secundários associados à redução cruenta da luxação de quadris artrogripóticos, em especial quando utilizada a via de acesso anteromedial, devido à subluxação progressiva ${ }^{(4,8,10,13)}$. Neste estudo, houve necessidade de procedimento secundário em dois quadris reduzidos através da via anteromedial. Staheli et $a l^{(11)}$ relataram melhores resultados em relação à amplitude de movimento nos quadris tratados com redução via anteromedial em relação aos submetidos à redução via iliofemoral devido à não realização de capsulorrafia.

Em nosso estudo, pudemos observar que, dos 10 quadris tratados com redução via anteromedial, todos ganharam amplitude de movimento, com oito bons resultados e dois com resultado regular devido à subluxação, porém, clinicamente assintomáticos.

É consenso na literatura que a luxação unilateral do quadril nas crianças portadoras de artrogripose com até dois anos de vida deva ser tratada para prevenir o desenvolvimento de obliquidade pélvica, discrepância de membros inferiores e, secundariamente, escoliose, sendo o procedimento de escolha a redução pelo acesso iliofemoral com encurtamento femoral proximal $^{(6,7,11-13)}$.

Alguns autores relatam bons resultados com a redução via anteromedial, recomendando esta via até os seis meses de vida, mesmo quando o acometimento for bilateral ${ }^{(5,10,11,14)}$.

Szöke et $a l^{(4)}$ recomendam redução cruenta via anteromedial em casos uni ou bilaterais entre os três e seis meses de idade, em combinação com outras correções cirúrgicas de contraturas congênitas de joelho e pés.
O manejo dos quadris dos pacientes com AMC portadores de luxação bilateral é bastante controverso $^{(11,13)}$, e as indicações do tratamento não são bastante claras.

Gibson e Urs ${ }^{(9)}$, Williams ${ }^{(10)}$ e Shapiro e Bresnan ${ }^{(21)}$ relatam que a luxação bilateral não deva ser tratada, porque na evolução os quadris não se mostram instáveis ou dolorosos, e após o tratamento cirúrgico pode-se desenvolver rigidez articular e dor. Gruel et $a l^{(13)}$ não tratam os quadris bilaterais em que o paciente não apresente prognóstico de marcha, fato com o qual também concordamos.

St. Clair e Zimbler ${ }^{(22)}$ indicam o tratamento da luxação bilateral para os pacientes que apresentam grau leve de acometimento dos membros superiores e boa amplitude de movimento articular dos quadris. Entretanto, alguns autores referem o tratamento com redução cirúrgica dos quadris luxados bilateralmente, independente do acometimento dos membros superiores $^{(4,5,11,13,14,22)}$.

Staheli et $a l^{(11)}$ reportaram 18 pacientes (24 quadris) com AMC e luxação de quadril. Os quadris tratados com redução cruenta por via anteromedial apresentam melhor mobilidade comparada àqueles tratados com redução incruenta ou redução cruenta via anterior iliofemoral, com resultados: bons em $80 \%$ dos casos; $12 \%$ regulares; e $8 \%$ ruins $^{(2,11)}$. Baseado em nosso estudo, bons resultados são obtidos quando o quadril luxado nos pacientes com AMC é tratado através do acesso anteromedial em crianças abaixo do primeiro ano de vida apresentando comprometimento unilateral ou bilateral.

No caso de crianças mais velhas que apresentam luxação unilateral, a redução cirúrgica associada ao encurtamento femoral é o tratamento de escolha ${ }^{(3)}$.

\section{CONCLUSÃO}

Apesar de ser uma amostra pequena de casos, observamos que em nenhum deles evoluiu com rigidez articular, que com esta técnica não houve redução da mobilidade articular e que foi possível manter o quadril centrado na maioria dos casos. Assim, consideramos a via anteromedial uma boa opção para o tratamento da luxação de quadril em pacientes no primeiro ano de vida em luxações uni ou bilaterais com artrogripose múltipla congênita e que apresentem potencial de marcha independente da função dos membros superiores. 


\section{REFERÊNCIAS}

1. Fisher RL, Johnstone WT, Fisher WH Jr, Goldkamp OG. Arthrogryposis multiplex congenita: a clinical investigation. J Pediatr. 1970;76(2):255-61.

2. Stern WG. Arthrogryposis multiplex congenita. JAMA. 1923;81:1507-10.

3. Bevan WP, Hall JG, Bamshad M, Staheli LT, Jaffe KM, Song K. Arthrogryposis multiplex congenita (amyoplasia): an orthopaedic perspective. J Pediatr Orthop. 2007;27(5):594-600.

4. Szöke G, Staheli LT, Jaffe K, Hall JG. Medial-approach open reduction of hip dislocation in amyoplasia-type arthrogryposis. J Pediatr Orthop. 1996;16(1):127-30.

5. Coleman SS. Teratologic congenital dislocation. In: Congenital dysplasia and dislocation of the hip. St. Louis: C.V. Mosby; 1978. p. 249-56.

6. Huurman WW, Jacobsen ST. The hip in arthrogryposis multiplex congenita. Clin Orthop Relat Res. 1985;(194):81-6.

7. Drumond DS, Siller TS, Cruess RL. Management of arthrogryposis multiplex congenital. Instr Course Lect. 1974;23:79-95.

8. Lloyd-Roberts GC, Letting AW. Arthrogryposis multiplex congenital. J Bone Joint Surg Br. 1970;52(3):494-508.

9. Gibson DA, Urs ND. Arthrogryposis multiplex congenita. J Bone Joint Surg Br.1970;52(3):483-93.

10. Williams PF. Orthopaedic management in childhood. London: Black Scientific; 1982.

11. Staheli LT, Chew DE, Eliot JS, Muscat VS. Management of the hip dislocation in children with arthrogryposis. J Pediatr Orthop. 1983;7:681-5.

12. Drummond DS, Mackenzie DA. Scoliosis in arthrogryposis multiplex congenita. Spine (Phila Pa 1976). 1978;3(2):146-51.
13. Gruel CR, Birch JG, Roach JW, Herring JA. Teratologic dislocation of the hip. J Pediatr Orthop. 1986;6(6):693-702.

14. Herring JA, Banta JV. Arthrogryposis. J Pediatr Orthop. 1988;8(3):353-5.

15. Hoffer MM, Feiwell E, Perry R, Perry J, Bonnett C. Functional ambulation in patients with myelomeningocele. J Bone Joint Surg Am. 1973;55(1):137-48.

16. Sharp IK. Acetabular dysplasia. The acetabular angle. J Bone Joint Surg Br. 1961;43(2):268-72.

17. Wiberg G. Studies on dysplastic acetabular and congenital subluxation of the hip joint: with special reference to the complication of osteoarthritis. Acta Chir Scand. 1939;83(Suppl 58): 1-135.

18. Ogden JA. Changing patterns of proximal femoral vascularity. J Bone Joint Surg Am. 1974;56(5):941-50.

19. Ogden JA. Treatment positions for congenital dysplasia of the hip. J Pediatr.1975;86(5):732-4

20. Frielander HL, Westin GW, Wood WL Jr. Arthrogryposis multiplex congênita. J Bone Joint Surg Am. 1968;50(1):89-119.

21. Shapiro F, Bresnan MJ. Orthopaedic management of childhood neuromuscular disease. Part I: Spinal muscular atrophy. J Bone Joint Surg Am. 1982;64(5):785-9.

22. St Clair HS, Zimbler S. A plan of management and treatment results in the arthrogrypotic hip. Clin Orthop Relat Res. 1985;(194):74-80.

23. Salter RB, Kostuik J, Dallas S. Avascular necrosis of the femoral head as a complication of treatment for congenital dislocation of the hip in young children: a clinical and experimental investigation. Can J Surg. 1969;12(1):44-61. 